Aims To identify the predictors of CD when presented with LD. Methods 215 LD patients had undergone prospective and systematic evaluation for $\mathrm{CD}$ and other recognised associations.

The gold-standard diagnosis of $\mathrm{CD}$ was based upon the presence of HLA-DQ2 and/or DQ8, persistence or progression of LD following a gluten challenge, symptomatic improvement on a gluten-free diet, and no alternate cause found.

Binary logistic regression models, adjusting for age and gender, were subsequently performed to compare presenting variables between $\mathrm{CD}$ and non-CD cases, and to determine their sensitivity, specificity, positive and negative predictive values (PPV and NPV).

Results CD was diagnosed in 47 cases $(22 \%)$ and non-CD in 168 cases $(78 \%)$. There was no statistical difference in demographics, clinical symptoms (i.e. diarrhoea, weight loss, abdominal pain), anaemia or haematinics between the $\mathrm{CD}$ and non-CD group.

Patients with $\mathrm{CD}$, in comparison to non-CD, were significantly more likely to have a positive family history of CD (21.3\% vs. $3.6 \%$, OR 6.81; PPV 62.5\%, NPV 81.4\%, specificity 96.4\%), positive HLA-DQ status (100\% vs. $49.4 \%$; PPV $36.2 \%$, NPV 100\%, specificity 50.6\%), and presence of endomysial antibody [EMA] (49\% vs. 0.6\%, OR 159; PPV 96\%, NPV 87\%, specificity 99.4\%); all $\mathrm{p} \leq 0.001$.

A normal tissue transglutaminase antibody (TTG) level was seen in $29.8 \% \mathrm{CD}$ and $82.7 \%$ non-CD cases (OR 0.086, p < 0.001; PPV 9.1\%). There was no difference in the prevalence of TTG levels 1-2 x upper limit of normal (ULN) between the groups (29.8\% CD vs. $14.3 \%$ non-CD; PPV 38\%). However, TTG levels between 3-20 x ULN were significantly more prevalent in the CD group ( $31.9 \%$ vs. $3 \%$; PPV $66.6 \%>87.5 \%)$, whilst a TTG $>20 \times$ ULN was exclusive to CD $(8.5 \%$, p < 0.001, PPV 100\%).

Conclusion At the outset, only the presence of positive EMA or TTG $>20 \times$ ULN are highly predictive and specific for CD. However, as they have limited sensitivities, most patients with LD require further work-up prior to diagnostic confirmation. Disclosure of Interest None Declared.

\section{PTU-152 PEPSIN IN SALIVA FOR THE DIAGNOSIS OF GASTRO-ESOPHAGEAL REFLUX DISEASE}

${ }^{1} \mathrm{JO}$ Hayat*, ${ }^{2} \mathrm{~S}$ Gabieta-Somnez, ${ }^{2} \mathrm{E}$ Yazaki, ${ }^{1} \mathrm{~J}-\mathrm{Y}$ Kang, ${ }^{3} \mathrm{~A}$ Woodcock, ${ }^{3} \mathrm{P}$ Dettmar, ${ }^{4} \mathrm{~J}$ Mabary, ${ }^{2} \mathrm{C}$ Knowles, ${ }^{2} \mathrm{D}$ Sifrim. ${ }^{1}$ Gastroenterology, St. George's, University of London, UK; ${ }^{2}$ Centre for Digestive Diseases, Barts and the London School of Medicine and Dentistry, London, UK; ${ }^{3}$ RD Biomed Limited, Hull, UK; ${ }^{4}$ Sandhill Sci., Colorado, USA

\subsection{6/gutjnl-2014-307263.226}

Introduction Current diagnostic tests for GORD have moderate sensitivity/specificity and can be invasive and expensive. Pepsin detection in saliva has been proposed as an "office-based" method for GORD diagnosis. The aims of this study were to establish normal values of salivary pepsin in a large cohort of healthy asymptomatic subjects and to determine its value to discriminate patients with reflux-related symptoms (GORD, hypersensitive oesophagus) from functional heartburn.

Methods 100 asymptomatic controls and 111 patients with heartburn underwent MII-pH monitoring and simultaneous salivary pepsin determination on waking, after lunch and dinner. Cut off value for pepsin positivity was $16 \mathrm{ng} / \mathrm{ml}$. Patients were divided into GORD (increased acid exposure time (AET) $\mathrm{n}=$ 58); Hypersensitive Oesophagus (HO) (normal AET and + SAP), $\mathrm{n}=26$ ) and Functional Heartburn $(\mathrm{FH})$ (normal AET and -
SAP, $\mathrm{n}=27$ ). Multiple group comparisons were performed using one-way ANOVA followed by with Tukey's Test for Gaussian distributed data and the Kruskall-Wallis Test with Dunns comparison for non-Gaussian data. Receiver Operator Characteristic curves were constructed to determine and compare the sensitivity and specificity of different pepsin cut-off concentrations.

Results $1 / 3$ of asymptomatic subjects had pepsin in saliva at low concentration $(0(0-59) \mathrm{ng} / \mathrm{ml})$. Patients with reflux-related symptoms (GORD and $\mathrm{HO}$ ) had higher prevalence $(77-89 \%)$ and pepsin concentration than controls (HO, 237(52-311) ng/ml and GORD, 121(29-252) ng/ml) ( $<$ 0.05). Patients with FH had low prevalence $(33 \%)$ and concentration of pepsin in saliva $(0$ $(0-40) \mathrm{ng} / \mathrm{ml})$. The area under the receiver operating characteristic curve had a value of $0.8034+/-0.04$ (95\% confidence interval 0.719 to $0.8873, \mathrm{p}<0.0001)$. A positive test had $77.6 \%$ sensitivity and $63.2 \%$ specificity for diagnosis of GORD/HO. When all saliva samples were negative, there was $80 \%$ probability that symptoms were not due to reflux $(\mathrm{FH})$. One positive sample with $>210 \mathrm{ng} / \mathrm{ml}$ pepsin suggested the presence of GORD/HO with $95 \%$ probability.

Conclusion In patients with symptoms suggestive of GORD, salivary pepsin can be used to confirm or reject the diagnosis before empirical PPI treatment. This may lessen the use of unnecessary anti-reflux therapy and the need for further invasive and expensive diagnostic methods.

Disclosure of Interest J. Hayat: None Declared, S. Gabieta-Somnez: None Declared, E. Yazaki: None Declared, J.Y. Kang: None Declared, A. Woodcock Employee of: RD Biomed Ltd, P. Dettmar Employee of: RD Biomed Ltd, J. Mabary Employee of: Sandhill Sci., C. Knowles: None Declared, D. Sifrim Grant/ research support from: Sandhill Sci.

\section{PTU-153 THE TIMING OF ONCOGENIC EVENTS IN THE EVOLUTION OF THE OESOPHAGEAL ADENOCARCINOMA GENOME AND IMPLICATIONS FOR CLINICAL DIAGNOSTICS}

${ }^{1} \mathrm{~J}$ Weaver* ${ }^{*}{ }^{2} \mathrm{C}$ Ross-Innes, ${ }^{3} \mathrm{~N}$ Shannon, ${ }^{3} \mathrm{~A}$ Lynch, ${ }^{3} \mathrm{M}$ Murtaza, ${ }^{3} \mathrm{~T}$ Forshew, ${ }^{3} \mathrm{M}$ Dunning, ${ }^{4} \mathrm{~T}$ Underwood, ${ }^{5} \mathrm{R}$ Hardwick, ${ }^{3} \mathrm{M}$ Eldridge, ${ }^{1} \mathrm{P}$ Edwards, ${ }^{3} \mathrm{~N}$ Rosenfeld, ${ }^{3} \mathrm{~S}$ Tavare, ${ }^{2} R$ Fitzgerald on behalf of The Occams, ICGC Oesophageal Adenocarcinoma consortium. ${ }^{1}$ Department of Pathology, Cambridge, UK; ${ }^{2}$ Hutchison-MRC, Cambridge, UK; ${ }^{3}$ CRUK Cambridge Institute, Cambridge, UK; ${ }^{4}$ Cancer Sciences Division, Southampton, UK; ${ }^{5}$ Addenbrooke's Hospital, Cambridge University NHS Foundation Trust, Cambridge, UK

\subsection{6/gutjil-2014-307263.227}

Introduction A series of clonal expansions are thought to underlie the progression of Barrett's oesophagus (BE) to oesophageal adenocarcinoma (OAC). Each expansion carries with it somatic driver mutation (s) fixing it within a larger population and therefore increasing the likelihood of acquiring a second mutation. However, the precise order in which somatic variants occur remains unknown.

Methods We performed whole genome sequencing in 25 cases of OAC and 3 matched cases of BE. Findings were validated in a larger cohort of OACs $(\mathrm{n}=90)$, metaplastic never-dysplastic BE (NDBE, $\mathrm{n}=66$ with a median follow-up of 58 months) and high-grade dysplasia $(n=43)$ using amplicon resequencing. Mutational signatures and gene-centric somatic mutations were determined using an in-house pipeline incorporating standard statistical methods and the publically available EMu pipeline.

Results There were 7 distinct mutational signatures present in both early (BE) and late disease (OAC). Fifteen genes were determined to be potential novel drivers of OAC development. 